\title{
Tuberculosis monoarticular de la muñeca. Comunicación de un caso
}

\author{
José Palma, Eduardo Díaz, Sergio Islas, Julio Silva y Sergio Mella
}

\section{Monoarticular tuberculosis of the wrist: a case report}

Monoarticular tuberculosis of the wrist is a rare presentation of primary tuberculosis, being more common skeletal forms involving the spine. Extraspinal tuberculous osteomyelitis is rare and comprises only 2 to $3 \%$ of all cases of osteoarticular Mycobacterium tuberculosis infections. We present a case of a 49 years old female patient, who worked as an hospital cleaning employed without other comorbidity. After a low energy injury of the wrist she suffered pain syndrome diagnosticated as a flexor tendinopathy, managed with nonsteroidal antiinflammatory drugs and physical therapy. Eight months later patient evolves with chronic pain in range of motion of right wrist joint, leading to a complete radiological, surgical biopsy and cultures. Histology, and molecular biology confirmed the wrist joint tuberculosis diagnosis. Pharmacological treatment and physical therapy were initiated with appropriated response.

Key words: Tuberculosis, wrist, joint pain, monoarticular arthritis.

Palabras clave: Tuberculosis, muñeca, dolor articular, monoartritis.

\section{Introducción}

$\mathrm{L}$

a tuberculosis (TBC) continúa siendo un problema de salud pública en el mundo debido a su incidencia en países en desarrollo, al aumento de infecciones por M. tuberculosis resistente a fármacos y su asociación con la infección por VIH/SIDA; así como a la dificultad diagnóstica en las presentaciones atípicas ${ }^{1}$. La TBC extrapulmonar representa cerca de 20 a $30 \%$ de los casos de esta enfermedad, según series publicadas en distintos paises ${ }^{2}$. La TBC esquelética u osteoarticular, a su vez representa una proporción muy baja, en relación al total de las infecciones causadas por M. tuberculosis. La localización más común es la TBC vertebral, con solo un 2 a $3 \%$ de compromiso extravertebral, siendo las articulaciones más afectadas la rodilla y la cadera ${ }^{3}$. La localización a nivel del carpo y de la muñeca es excepcional, pudiendo aparecer de forma aislada o asociada a un compromiso visceral en 20 a $40 \%$ de los casos ${ }^{4}$.

Las manifestaciones clínicas características incluyen dolor, tumefacción, pérdida del balance articular e impotencia funcional. Dado lo infrecuente de la localización aislada de la muñeca, presentamos el caso clínico de una paciente con primer diagnóstico de un tumor de muñeca y que tenía, entre otros antecedentes, trabajar en un hospital.

\section{Caso clínico}

Paciente de sexo femenino 49 años, auxiliar del servicio clínico de urgencias, con 20 años de experiencia en su oficio y 10 en el servicio de urgencias. Dentro de sus antecedentes destacaban solo tabaquismo ocasional, sin uso de medicamentos en forma crónica, residencia urbana, sin condiciones de hacinamiento, con serología negativa para hepatitis B al iniciar sus labores, sin screening para otras patologías. Su mano dominante era la derecha. Tenía el antecedente de un cuadro, de ocho meses de evolución, de dolor en la muñeca derecha que inició, según su relato, luego de movilizar una camilla. El dolor se intensificaba al realizar actividades cotidianas, empeorando en forma progresiva. Consultó en un centro de atención primaria, donde se diagnosticó una tendinopatía flexora de la muñeca derecha que se manejó con analgesia, reposo y fisioterapia. La paciente evolucionó en forma tórpida, con una recuperación parcial y posterior recidiva de su sintomatología inicial, agregándose rigidez, disminución de la fuerza de prensión con limitación laboral y de las actividades de la vida cotidiana. Ante la persistencia de sus molestias, acudió a policlínico de traumatología nueve meses después de su primera consulta. En dicha ocasión, destacó en el examen físico, dolor a la movilización de la muñeca derecha, mayor en flexión y con disminución significativa de los rangos articulares. El examen neurovascular estaba conservado, sin signos de alteración de las funciones parasimpáticas.

Se solicitaron radiografías de muñeca anteroposterior y lateral que no evidenciaron mayores alteraciones (Figuras 1 y 2). Se solicitó una ecotomografía de muñeca derecha que mostró una imagen redondeada anecogénica, con refuerzo posterior, que medía $5 \times 4 \times 3 \mathrm{~mm}$ en sus ejes
Hospital Traumatológico de Concepción, Chile. Universidad de Concepción Departamentos de Cirugía y Medicina Interna, Concepción, Chile.

Conflictos de interés: sin conflictos de interés. Fuente de financiamiento: Sin financiamiento externo.

Recibido: 24 de abril de 2017 Aceptado: 11 de septiembre de 2017

Correspondencia a: José Palma C. jose.palma.concha@gmail.com 
principales (Figura 3). La resonancia magnética (RM) mostró una lesión quística, de similares características a la observada en ecotomografía, que desplazaba el nervio mediano hacia cubital y con evidencia de compromiso de la fascia volar, sin trayectos fistulosos (Figura 4).

Se realizó una intervención quirúrgica con la hipótesis
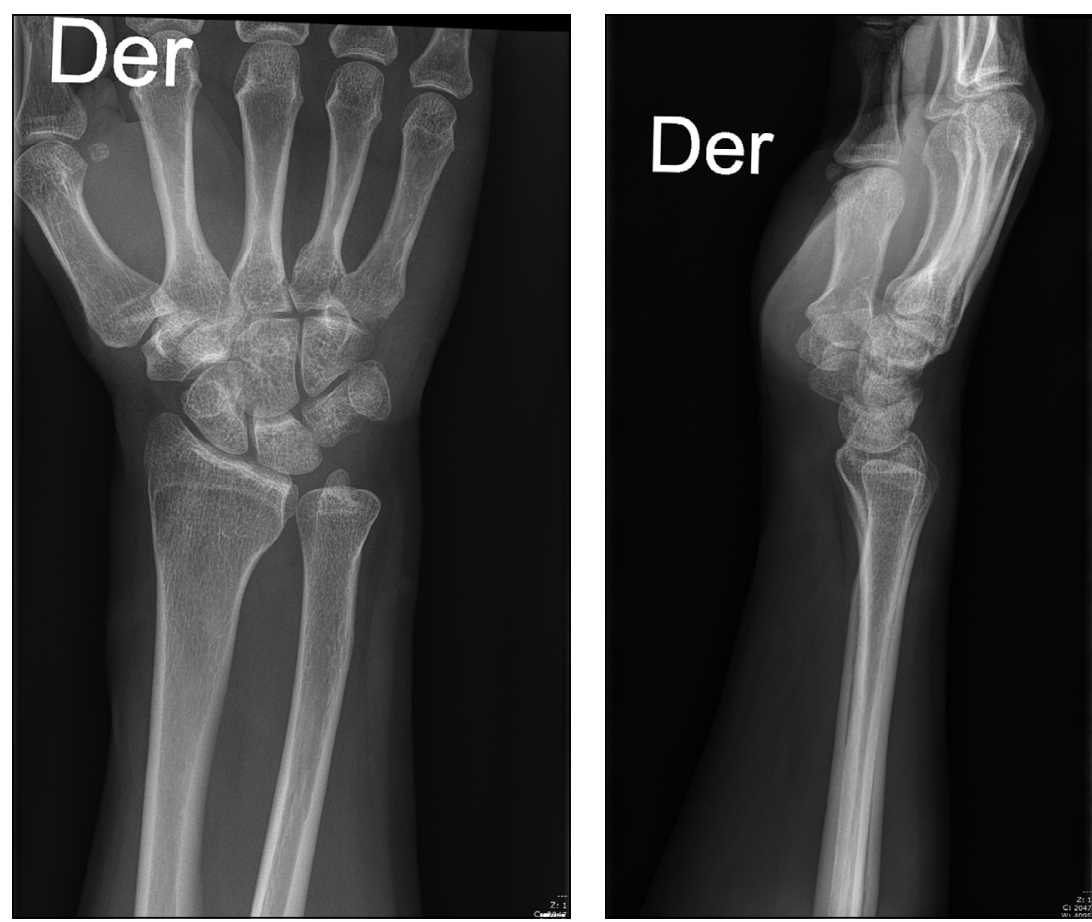

Figuras 1 y 2. Radiografías de muñeca anteroposterior y lateral; respectivamente, que no evidencian afectación ósea ni lesiones adyacentes.

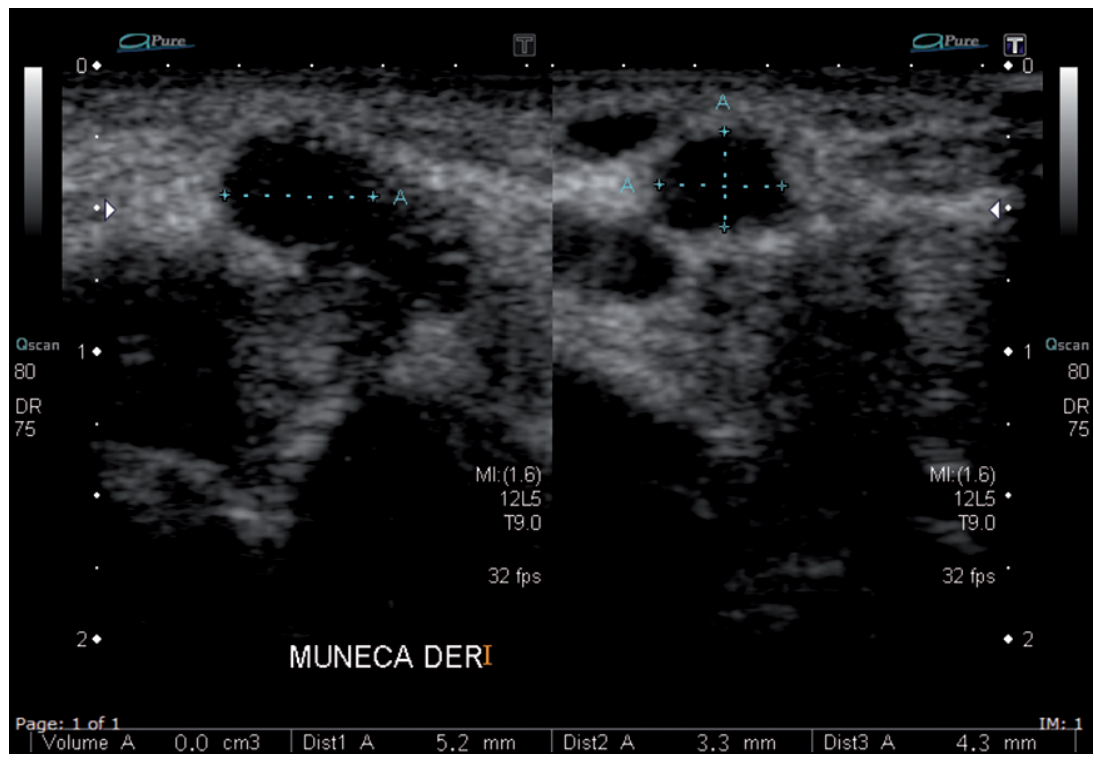

Figura 3. Ecotomografía de partes blandas: muñeca derecha. Muestra imagen redondeada anecogénica, con refuerzo posterior, que mide $5 \times 4 \times 3 \mathrm{~mm}$ en sus ejes principales. diagnóstica de un tumor de tejidos blandos de muñeca derecha. Durante el procedimiento se tomaron muestras de tejidos en los planos superficiales y profundos para histología, cultivo para aerobios y micobacterias, junto con una RPC para M. tuberculosis. La histología con fijación en formalina y tinción hematoxilina/eosina demostró la presencia de granulomas epiteloideos de aspecto tuberculoso en el tejido subcutáneo fibroadiposo, sinovial con extensa inflamación crónica granulomatosa de aspecto tuberculoídeo y una sinovitis hiperplásica reactiva. La histoquímica con tinciones de auramina y Ziehl-Neelsen resultaron negativas para bacilos alcohol ácido resistentes y la tinción de Grocott fue negativa para hongos. El cultivo para aerobios de tejidos superficiales y profundos fue negativo. La RPC GeneXpert-RIF resultó positiva para $M$. tuberculosis, y finalmente el cultivo específico para $M$. tuberculosis, con método de Petroff modificado, informó la presencia de 30 colonias típicas a los 30 días de observación.

Dentro del estudio de diseminación se realizaron una radiografía de tórax que fue normal y exámenes generales de laboratorio que estaban dentro de rangos aceptables, donde sólo destacaba una leve elevación de la VHS, con un valor de $21 \mathrm{~mm} / \mathrm{h}$, una proteína $\mathrm{C}$ reactiva de $0,3 \mathrm{mg} / \mathrm{L}$ y leucocitos de $7.890 / \mathrm{mm}^{3}$, con una fórmula leucocitaria normal. Las baciloscopias de expectoración con técnica de Ziehl-Neelsen fueron informadas como negativas para la presencia de bacilos ácido-alcohol resistentes, en 100 campos observados. La serología de VIH fue negativa. Se inició estudio de los contactos intradomiciliarios (el marido y dos hijos mayores de 15 años, que se encontraban asintomáticos) con baciloscopias de expectoración y cultivos para $M$. tuberculosis negativos y radiografías de tórax normales.

Con los resultados histológicos y de la biología molecular de tejido (posteriormente confirmados por cultivos) en una paciente sin síntomas respiratorios, con radiografía de tórax normal y baciloscopias negativas, se hizo el diagnóstico de una TBC extrapulmonar con localización aislada en la muñeca derecha, por lo que recibió tratamiento anti-tuberculoso con isoniacida, rifampicina, pirazinamida y etambutol según normas del programa nacional de TBC.

Con la terapia anti-tuberculosa y fisioterapia, la paciente evolucionó favorablemente. Se realizó una evaluación con score de la Clínica Mayo para determinar funcionalidad de articulación de muñeca, progresando desde 50 puntos (función pobre de la muñeca) al inicio hasta 80 puntos (buena función de la muñeca) luego de cinco meses de tratamiento.

La RM de control, a los cinco meses de evolución, mostró una involución significativa en el tamaño de la lesión quística y del proceso inflamatorio global de la articulación de la muñeca (Figura 5). 


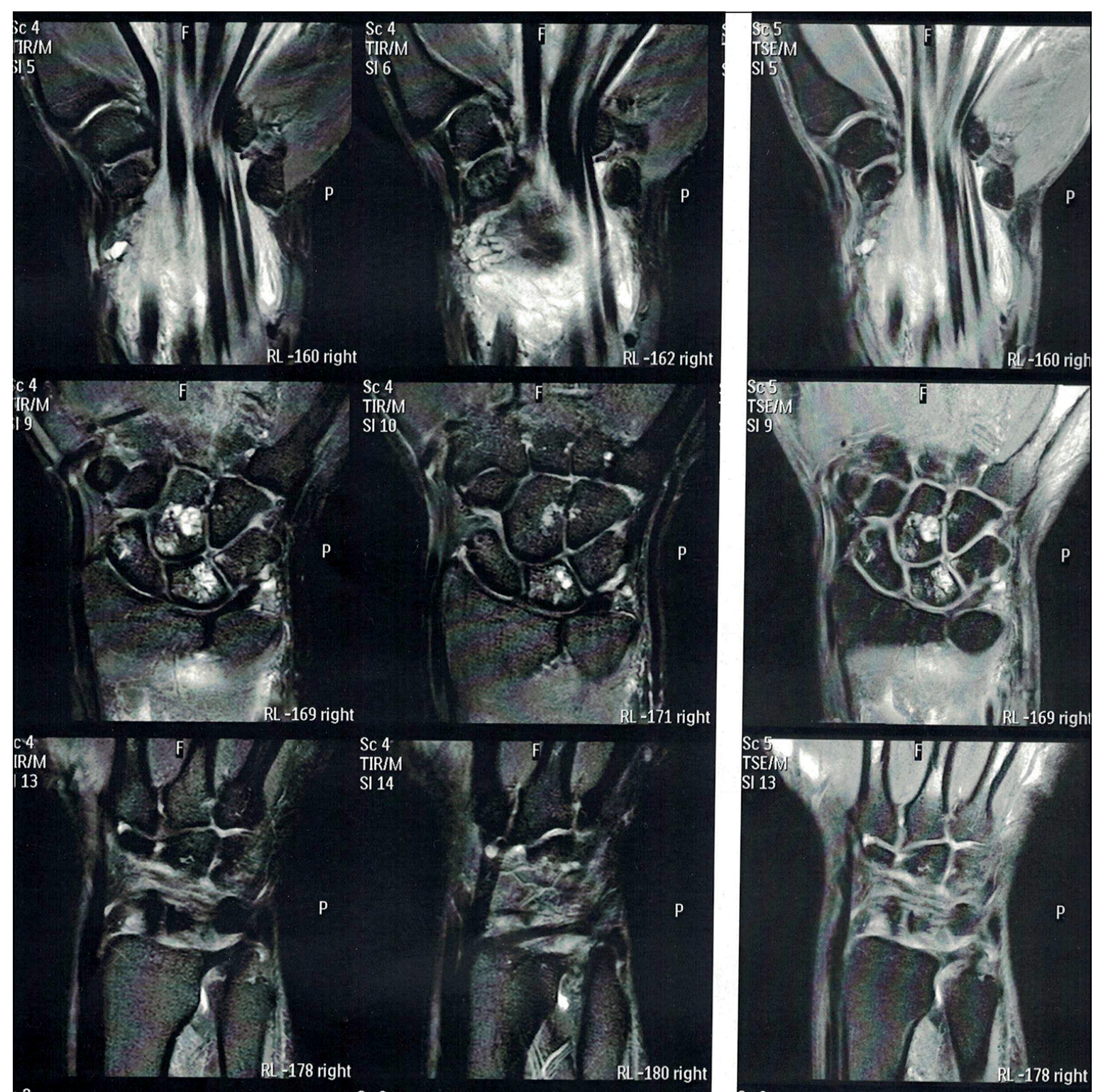

\section{Discusión}

Figura 5. Resonancia magnética de control, solicitada 5 meses luego de iniciado el tratamiento, que muestra involución de la lesión quística descrita en margen radial distal de la muñeca derecha, junto con disminución significativa del proceso inflamatorio global de la articulación radio-cubito-carpiana. Imágenes nodulares residuales en huesos del carpo (grande, semilunar).
Las presentaciones atípicas e infrecuentes de la TBC se mantienen aún como un desafío diagnóstico. Así, en el caso clínico presentado se planteó como primera hipótesis una lesión tumoral. En países como el nuestro debe mantenerse una conducta de sospecha clínica en una amplia variedad de presentaciones, particularmente cuando existe un factor epidemiológico de exposición, como la paciente presentada ${ }^{5,6}$.

El personal de salud (PS) es afectado en forma transversal. Los servicios de urgencia generalmente no cuentan con infraestructura adecuada, existe hacinamiento de pacientes, con dificultad en lograr una hospitalización
Figura 4. Resonancia magnética de muñeca derecha, evidencia lesión quística de $5 \times 3 \times 3 \mathrm{~mm}$ en sus ejes principales, en margen radial distal de muñeca derecha, con compromiso y desplazamiento del nervio mediano hacia cubital y extensión del proceso hacia fascia palmar. 
precoz en salas generales y prácticamente no existen habitaciones individuales con presión negativa. Por otra parte, se ha demostrado que el nivel socio-económico está asociado con este riesgo, observándose una mayor frecuencia de casos entre PS de menores ingresos ${ }^{6}$. Si bien la mayor parte de los casos de TBC detectados en PS son pulmonares, es importante tener en cuenta otras formas de presentación de la enfermedad, más aun en casos oligosintomáticos.

La presentación osteoarticular de la TBC es extremadamente infrecuente. Su etiopatogenia corresponde generalmente a una diseminación hematógena. Con menos frecuencia puede ser originada por una diseminación linfática retrógrada o por contiguidad desde sitios con bacilos activos?

Respecto a la presentación clínica de la TBC de la muñeca, la formación de abscesos puede encontrarse en alrededor de $20-25 \%$ de los casos, presentándose trayectos fistulosos a dorsal y volar en la mano. La sintomatología clásica de TBC diseminada, (diaforesis nocturna, astenia $\mathrm{y}$ anorexia) es inconstante $(25-40 \% \text { de los casos })^{4}$.

Se necesita un alto nivel de sospecha clínica para situar a esta forma de TBC dentro del diagnóstico diferencial de una monoartritis. Con respecto a las pruebas de laboratorio puede encontrarse un aumento de reactantes de fase aguda (VHS y PCR). La citología del líquido articular en general se caracteriza por una hipercelularidad superior a 50.000 con predominio en $80 \%$ de los casos de neutrofilos.

En el estudio radiológico se describe la tríada de Phemister en proyecciones anteroposterior y lateral de muñeca que involucra osteoporosis yuxtaarticular, erosiones óseas periféricas y pinzamiento de la interlinea ${ }^{8}$.

En casos muy avanzados puede encontrarse una osteolisis multigeódica en todos los huesos del carpo. La gammagrafía ósea con Tc-99 tiene un rol en descartar el compromiso poliarticular. La RM y TC son útiles para la observación de abscesos, secuestros intraarticulares o realizar punciones guiadas.

Entre los diagnósticos diferenciales se encuentran las osteomielitis crónicas, sinovitis villonodular, sarcoidosis, hiperparatiroidismo, enfermedad de Paget y artrosis postraumática. Así la TBC esquelética puede "mimetizarse" con muchas otras entidades, retardando su diagnóstico".

El diagnóstico de certeza de la TBC osteoarticular requiere un estudio histológico, donde pueden encontrarse granulomas de células epiteloides y células gigantes tipo Langhans asociado a una necrosis caseosa ${ }^{10}$.

El diagnóstico microbiológico es más inconstante siendo la baciloscopia y el cultivo en medio de Lowenstein-
Jensen frecuentemente negativos

En el caso clínico presentado, una clínica oligosintomática con imágenes no concluyentes y exámenes de laboratorio sin grandes alteraciones, hicieron plantear la necesidad de realizar una biopsia quirúrgica de las lesiones. Los hallazgos histopatológicos encontrados fueron finalmente claves para el diagnóstico de una TBC articular de muñeca, el que fue confirmado posteriormente por biología molecular y cultivos para M. tuberculosis.

El cultivo para $M$. tuberculosis realizado por el método de Petroff modificado presenta una especificidad de 9599\% para la detección del bacilo y las técnicas de biología molecular, tienen una sensibilidad entre 61 y $96 \%$ en presentaciones extrapulmonares ${ }^{11}$. En el caso presentado, la positividad de las pruebas de biología molecular permitieron el inicio precoz de la terapia anti-tuberculosa en la paciente, asi como el estudio de sus contactos.

Con respecto al tratamiento de este tipo de TBC, debe hacerse énfasis a que sigue siendo principalmente médico ${ }^{5}$ la cirugía tiene un papel limitado reservándose para la realización de biopsias, fistulectomías, drenaje de abscesos, tenosinovectomías y artrodesis en casos muy avanzados con gran deterioro articular ${ }^{12}$. El pronóstico depende de la articulación afectada y el daño producido al momento del diagnóstico, siendo variable de acuerdo a las series publicadas, existiendo un número limitado de publicaciones al respecto ${ }^{10,12}$.

\section{Resumen}

La tuberculosis monoarticular aislada de la muñeca es una forma infrecuente de presentación de esta enfermedad, siendo más común el compromiso vertebral. Las formas extravertebrales representan sólo 2 a $3 \%$ de las infecciones óseas por Mycobacterium tuberculosis. Presentamos el caso clínico de una mujer de 49 años, con antecedentes de trabajar en labores de aseo en un hospital, que posterior a un trauma de baja energía evolucionó con un cuadro de dolor en la articulación de la muñeca derecha. Diagnosticada inicialmente como una tendinopatía flexora, recibió tratamiento con antiinflamatorios y fisioterapia. Ocho meses después la paciente continuó con dolor a la movilización por lo que se realizó un estudio imagenológico, biopsia y cultivos de tejido óseo. El estudio histopatológico y de biología molecular del tejido confirmó una tuberculosis de muñeca derecha. Se trató con terapia anti-tuberculosa y fisioterapia, consiguiéndose la recuperación funcional de la muñeca. 


\section{Referencias bibliográficas}

1.- World Health Organization. Global Tuberculosis Control: Surveillance, Planning, Financing. WHO Report 2002. Ginebra, Suiza. Librería WHO en linea. 2002: 3-27. Fecha de acceso: 24 de abril de 2017. Disponible en: http://www.hst.org.za/sites/default/files/ GlobalTB_2002.pdf

2.- Wares F, Balasubramanian R, Mohan A, Sharma S. Tuberculosis Control in India. Directorate General of Health Services. Ministry of Health and Family Welfare. New Delhi. India. Elsevier. 2005: 95-114. (Fecha de acceso: 24 de abril de 2017). Disponible en: https://pdfs.semanticscholar.org/0eaf/20ddbffe4 140add6ed37ba913e27d196e7c0.pdf

3.- Culqui-Lévano D R, Rodríguez-Valín E, Donado-Campos J M. Analysis of extrapulmonary tuberculosis in Spain: 20072012 National Study. Enferm Infecc Microbiol Clin 2017; 35: 82-7.
4.- Torres Lozano P, Gallach Sanchis D, Pardo Coello M M. Tuberculosis osteoarticular de muñeca. Artritis destructiva de muñeca secundaria a tuberculosis extrapulmonar. Rev Esp Cir Ortop Traumatol 2012; 56: 378-80.

5.- Herrera T, Torres Z, Arias F, Peña C, Pickett S, Lepe R, et al. Programa nacional de control y eliminación de la tuberculosis. Normas técnicas para el control y la eliminación de la tuberculosis. Subsecretaría de Salud Pública. División de Prevención y Control de Enfermedades. Ministerio de Salud. Santiago de Chile. 2014:17-36. (Fecha de acceso: 24 de abril de 2017). Disponible en: http://web.minsal.cl/sites/default/files/ NORMA_TECNICA_TUBERCULOSIS.pdf

6.- Fica A, Cifuentes M, Ajenjo M, Jemenao M, Zambrano A, Febré N, et al. Tuberculosis en personal de salud. Rev Chilena Infectol 2008; 25: 243-55.

7.- N A, Ahmad F, Huda N. Osteoarticular tuberculosis-a three years' retrospective study. J Clin Diagn Res 2013; 7: 2189-92.

8.- Yao D C, Sartoris D J. Musculoskeletal tuberculosis. Radiol Clin North Am 1995; 33 : 679-89.

9.- Magnussen A, Amirthanayagam T, Sofat R Osteoarticular tuberculosis: The great mimicker still catches us out-a case report. Acta Orthop 2016; 87: 83-4.

10.- Sunderamoorthy D, Gupta V, Bleetman A. TB or not TB: an unusual sore finger. Emerg Med J 2001; 18: 490-1.

11.- Drobniewski F, Nikolayevskyy V, Maxeiner H, Balabanova Y, Casali N, Kontsevaya I, et al. Rapid diagnostics of tuberculosis and drug resistance in the industrialized world: clinical and public health benefits and barriers to implementation. BMC Med 2013; 11: 190

12.- Dlimi F, Bellarbi S, Mahfoud M, Berrada M, El Bardouni A, El Yaacoubi M. Tuberculosis of the hand and wrist: different aspects about 30 cases. Chir Main 2011; 30: 198-204. 